\title{
Dietrich, Cornelie
}

\section{Vorderbühne - Hinterbühne. Zur Interdependenz der Horizonte von Diversität und Gleichheit}

Hedderich, Ingeborg [Hrsg.]; Reppin, Jeanne [Hrsg.]; Butschi, Corinne [Hrsg.]: Perspektiven auf Vielfalt in der frühen Kindheit. Mit Kindern Diversität erforschen. 2., durchgesehene Auflage. Bad Heilbrunn : Verlag Julius Klinkhardt 2021, S. 60-75

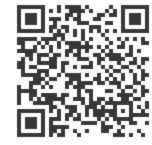

Quellenangabe/ Reference:

Dietrich, Cornelie: Vorderbühne - Hinterbühne. Zur Interdependenz der Horizonte von Diversität und Gleichheit - In: Hedderich, Ingeborg [Hrsg.]; Reppin, Jeanne [Hrsg.]; Butschi, Corinne [Hrsg.]:

Perspektiven auf Vielfalt in der frühen Kindheit. Mit Kindern Diversität erforschen. 2., durchgesehene Auflage. Bad Heilbrunn : Verlag Julius Klinkhardt 2021, S. 60-75 - URN:

urn:nbn:de:0111-pedocs-222485 - DOI: 10.25656/01:22248

https://nbn-resolving.org/urn:nbn:de:0111-pedocs-222485

https://doi.org/10.25656/01:22248

in Kooperation mit / in cooperation with:

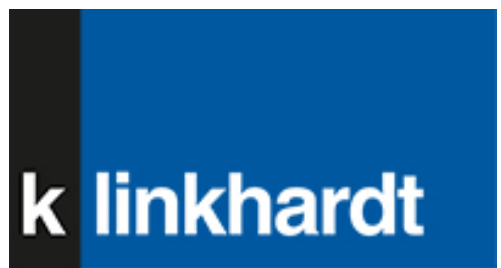

http://www.klinkhardt.de

\section{Nutzungsbedingungen}

Dieses Dokument steht unter folgender Creative Commons-Lizenz: http://creativecommons.org/licenses/by-nc-nd/4.0/deed.de - Sie dürfen das Werk bzw. den Inhalt unter folgenden Bedingungen vervielfältigen, verbreiten und öffentlich zugänglich machen: Sie müssen den Namen des Autors/Rechteinhabers in der von ihm festgelegten Weise nennen. Dieses Werk bzw. dieser Inhalt darf nicht für kommerzielle Zwecke verwendet werden und es darf nicht bearbeitet, abgewandelt oder in anderer Weise verändert werden.

Mit der Verwendung dieses Dokuments erkennen Sie die Nutzungsbedingungen an.

\section{Terms of use}

This document is published under following Creative Commons-License: http://creativecommons.org/licenses/by-nc-nd/4.0/deed.en - You may copy, distribute and transmit, adapt or exhibit the work in the public as long as you attribute the work in the manner specified by the author or licensor. You are not allowed to make commercial use of the work or its contents. You are not allowed to alter, transform, or change this work in any other way.

By using this particular document, you accept the above-stated conditions of use.

\section{Kontakt / Contact:}

\section{peDOCS}

DIPF | Leibniz-Institut für Bildungsforschung und Bildungsinformation

Informationszentrum (IZ) Bildung

E-Mail: pedocs@dipf.de

Internet: www.pedocs.de

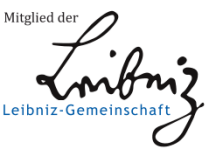




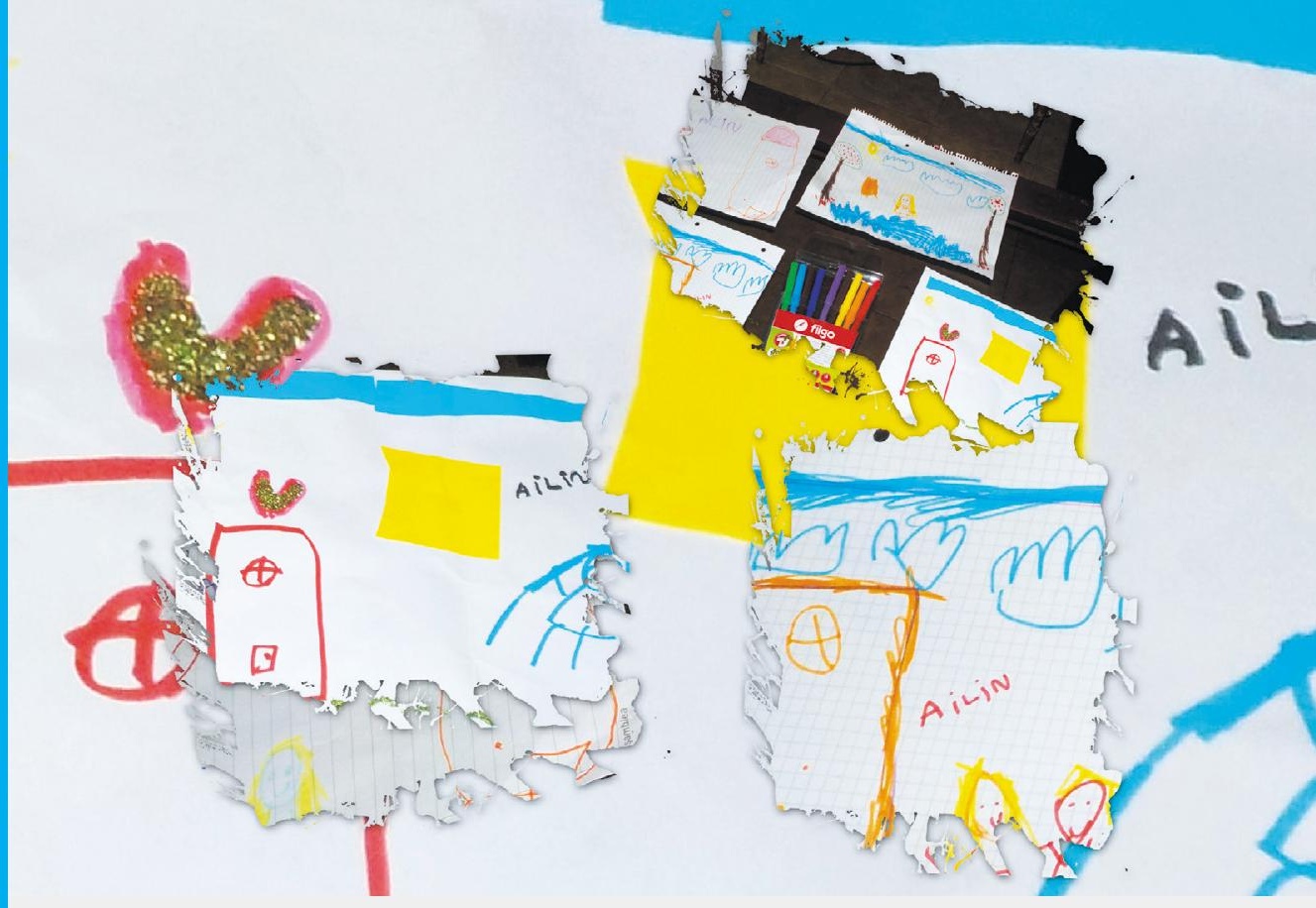

Ingeborg Hedderich

Jeanne Reppin

Corinne Butschi

(Hrsg.)

\section{Perspektiven auf Vielfalt in der frühen Kindheit}

Mit Kindern Diversität erforschen

2. Auflage 


\section{Ingeborg Hedderich Jeanne Reppin \\ Corinne Butschi \\ (Hrsg.)}

\section{Perspektiven auf Vielfalt in der frühen Kindheit}

Mit Kindern Diversität erforschen

2., durchgesehene Auflage 
Dieser Titel wurde in das Programm des Verlages mittels eines Peer-Review-Verfahrens aufgenommen. Für weitere Informationen siehe www.klinkhardt.de.

Bibliografische Information der Deutschen Nationalbibliothek Die Deutsche Nationalbibliothek verzeichnet diese Publikation in der Deutschen Nationalbibliografie; detaillierte bibliografische Daten sind im Internet abrufbar über http://dnb.d-nb.de.

2021.g. () by Julius Klinkhardt.

Foto Umschlagseite 1: Ailin, 5 Jahre, Provinz Entre-Rios, Argentinien und Corinne Butschi.

Druck und Bindung: Bookstation GmbH, Anzing.

Printed in Germany 2021.

Gedruckt auf chlorfrei gebleichtem alterungsbeständigem Papier.

(c) (1) $\Theta(D i e$ Publikation (mit Ausnahme aller Fotos, Grafiken und Abbildungen) ist veröffentlicht unter der Creative Commons-Lizenz: CC BY-NC-ND 4.0 International https://creativecommons.org/licenses/by-nc-nd/4.0/ 


\section{Inhalt}

Perspektiven auf Vielfalt in der frühen Kindheit.

Mit Kindern Diversität erforschen

Einleitung

Ingeborg Hedderich, Jeanne Reppin und Corinne Butschi

\section{Theorie}

Kindheit und Kindheitsforschung im Wandel

Corinne Butschi und Ingeborg Hedderich.

Erziehungswissenschaftliche Perspektiven auf Vielfalt, Heterogenität,

Diversity/Diversität, Intersektionalität

Katharina Walgenbach.

Vorderbühne - Hinterbühne. Zur Interdependenz der Horizonte von Diversität und Gleichheit

Cornelie Dietrich. 60

Differenzen und die Heterogenität von Kindern -

Einsätze blicktheoretischer Forschung

Friederike Schmidt.

\section{Methodologie und Methoden}

Kindgerecht forschen. Ein Überblick

Corinne Butschi und Ingeborg Hedderich.

Ankerpunkte, Wegmarken und Herausforderungen einer ethischen Forschung mit Kindern

Jeanne Reppin

Mit Kindern Interviews führen: Ein praxisorientierter Überblick

Susanne Vogl

Es ist noch jemand mit uns hier.

Puppet-Interviews in der Forschung mit Kindern

Marion Weise

Forschungsmethodische Vielfalt. Der Mosaic Approach

Sandra Schütz und Eva Theresa Böhm

Warum eigentlich? Philosophieren mit jungen Kindern

Martina Bernasconi. 


\section{Forschungszusammenhänge und Praxisreflexionen}

Ethnologische Kindheitsforschung im Überblick mit besonderer

Berücksichtigung der teilnehmenden Beobachtung

Werner M. Egli.....

Diversitätsbewusstes Denken und Handeln in Kindertageseinrichtungen -

Forschen mit Interviews und Interviewstreifzügen

Steffen Brockmann

Zur Relevanz der Zurechnung von Komplexität. Das Interview als

Methode der Datenerhebung im Alter früher Kindheit im Kontext von Behinderung

Martina Kaack.

Kamerakids: Forschen mit Photovoice

Corinne Butschi, Melike Hocaoglu, Manuel Zanardini, Valentin Mettler,

Ana Luisa Baumann-Santiago Martínez, Guillermina Chabrillon und

Ingeborg Hedderich.

Vielfalt in internationaler Zusammenarbeit erforschen. Ein Dialog

Corinne Butschi und Guillermina Chabrillon....

Einblick in ein vielfältiges Tätigkeitsfeld frühkindlicher Förderung in

Argentinien

Barbara Schoch

Das Erleben von Kindern mit Fluchterfahrung.

Forschen mit non-verbalen Zugängen

Marion Weise, Marion Lempp und Regine Morys

Wie erleben platzierte Vorschulkinder die Zugehörigkeit zu ihren

komplexen Beziehungswelten?

Forschen mit dem Geschichtenstammverfahren der

MacArthur Story Stem Battery

Maria Mögel.....

Lebenswelt Gemeinde: Mit Kindern forschen -

Aus Perspektiven und Methoden ein Mosaik zusammenfügen

Gabriela Muri und Heidi Simoni

Vom Schweizer Kindergarten ins Außerschulische, nach Ghana, und wieder zurück: Wenn Kinder und eine Ethnografin gemeinsam

,Grenzen' überschreiten

Ursina Jaeger

Die Autor*innen 
60

Cornelie Dietrich

\section{Vorderbühne - Hinterbühne. Zur Interdependenz der Horizonte von Diversität und Gleichheit}

In der inzwischen breit ausdifferenzierten Forschung zur Heterogenität in pädagogischen Feldern, deren Entstehung, Reproduktion und daraus erwachsenen pädagogisch-praktischen Herausforderungen, bleibt die theoretische und auch empirische Erforschung der Homogenitäts-Vorstellungen, -erwartungen, -fiktionen und -praktiken noch blass. Sie aber ist - neben den Antworten der Individualisierung und Inklusion - die Hintergrundfolie, auf die die aktuellen Debatten um einen angemessenen Umgang mit Heterogenität zu beziehen wären.

Der Text rekonstruiert zunächst drei verschiedene erziehungswissenschaftliche Zugänge zu Homogenitäts- und Gleichheitsvorstellungen, die in den Diskussionen um Heterogenität, Vielfalt und soziale Differenz wirksam sind. Die Inkommensurabilität der verschiedenen Argumentationen resultiert u.a. aus einer Verschränkung von gerechtigkeitstheoretisch-normativen und kulturell-deskriptiven Rahmungen im erziehungswissenschaftlichen Feld. Die daraus entstehenden Spannungen werden in einem nächsten Schritt diskutiert und an das Konzept interner Reflexivität von Gleichheit (vgl. Menke 2004) angeschlossen. Gegenüber den theoretischen Debatten werden im abschließenden Teil einige Desiderata praxeologischer Erforschung von Prozessen der Homogenese dargestellt.

\section{Einleitung}

Aus einer allgemeinpädagogischen Perspektive heraus erstaunt, mit welcher Selbstverständlichkeit man sich in der Pädagogik der frühen Kindheit des Themas Diversität inzwischen annimmt. Denn es ist noch nicht so lange her, dass zu den Strukturunterschieden zwischen Grundschule und Kindertagesstätten die Sozialintegrität gegenüber der Sozialselektivität gezählt wurde (vgl. Diehm 2008). Solange dies galt, so könnte man vermuten, braucht es keine wissenschaftliche Beschäftigung mit Diversität in Einrichtungen der Frühen Kindheit, da hier zunächst einmal ,keine Unterschiede gemacht ` werden - eine Ausnahme bilden die Debatten zur Geschlechtersozialisation. Die Thematisierung und wissenschaftliche Untersuchung von Unterschieden ist in der Erziehungswissen- 
schaft in der Regel verbunden mit der Thematisierung von Legitimationsweisen ihrer hierarchischen Ordnung. In der schulbezogenen Pädagogik ist damit eine Legitimierung von Platzierung und Selektion nach dem ersten Prinzip der Leistungsperformanz in Bezug auf schulische Leistungserwartungen verbunden. Alle anderen Differenzkategorien werden darauf bezogen. Erst wenn auch die frühpädagogischen Einrichtungen der selektiven Logik der Schule folgen, ihre Adressat*innen nach Differenzkategorien wie Leistung, Sprachvermögen, Migrationsstatus und soziokulturelle Herkunft zu differenzieren und damit hierarchisch zu platzieren oder eine solche Platzierung vorzubereiten, entsteht auch die Sorge um eine ungerechte, nicht legitimierbare Platzierung. Die beginnende Konjunktur des Diversitätsthemas in der Frühpädagogik legt also mehrere Lesarten nahe: Zum einen kann sie als Ausdruck dafür verstanden werden, dass auch schon in den Feldern Früher Bildung von allen Akteuren sehr wohl Unterschiede gemacht, hegemoniale Verhältnisse reproduziert werden und damit der Auftrag an eine wissenschaftliche Beschäftigung mit den modi operandi gegeben ist. Zum anderen lässt sie sich aber auch verstehen als Ausdruck einer zunehmenden Ausweitung schulischer Logik in die Frühe Kindheit hinein. Es entstehen dann andere Forschungsfragen, z.B. solche, die die Unterschiede zwischen Kindern vor allem als auszugleichende Unterschiede im Hinblick auf einen ,gelingenden' Schulbeginn untersuchen, etwa hinsichtlich des Sprachniveaus. In beiden Lesarten stehen je unterschiedliche Konzeptionen von Gleichheit Pate, die aber selten mitdiskutiert werden. Ich möchte daher im Folgenden primär nach diesen Vorstellungen von Homogenität und Gleichheit fragen. Denn beide Begriffshorizonte sind wechselseitig aufeinander verwiesen, sie befragen sich implizit gegenseitig, derjenige der Gleichheit und Homogenität jedoch wird in neueren erziehungswissenschaftlichen Debatten kaum einmal ausbuchstabiert, jedenfalls nicht im Lichte der Heterogenitätsforschung (vgl. etwa Drerup \& Fölling 2006; Liebau und Zirfas 2008). Gegenüber einer sehr beachtlichen Ausdifferenzierung des Forschungsfeldes um die Reproduktion sozialer Ungleichheit und die doing-difference Prozesse in den Institutionen von Bildung und Erziehung (vgl. z.B. Diehm et al. 2013; sowie die Sammelbände von Budde 2013; Koller, Casale \& Ricken 2014) bleibt in den erziehungswissenschaftlichen Debatten eigentümlich marginal die Frage, auf welche Vorstellungen von Gleichheit und Homogenität sich all die Differenz- und Heterogenitätsforschung und sie umgebenden Debatten beziehen und ob bzw. wie solche Vorstellungen sich im pädagogischen Feld verwirklichen. Gleichzeitig lässt sich auf der Ebene von Bildungsorganisation und -steuerung eine spannungsvolle Entwicklung von zunehmender Standardisierung einerseits und intensivierter Individualisierung andererseits beobachten. Beide 'Strategien ' beanspruchen, als eine angemessene Antwort auf die unübersichtlicher werdende Heterogenität gelten zu können, ohne dass ihre unterschiedlichen Referenzrahmen hinreichend systematisiert und damit transparent diskutierbar wären. 
Dass aber Heterogenität und Ungleichheit Kontur und Prägnanz gewinnen können nur im Horizont von Gleichheitsannahmen, ist nicht nur ein moralisches, rechtliches und damit handlungstheoretisch wirksames, sondern schon ein basales logisches Problem: Ungleichheit gibt es nicht an sich, sondern immer nur relational zwischen (mindestens) zwei Vergleichsdingen, die im Hinblick auf ein Drittes als entweder gleich oder verschieden erkannt, beschrieben oder produziert werden, so jedenfalls die Argumentation in der politischen Philosophie (vgl. Pauer-Studer 2000, S. 24ff.; Menke 2004).

Doch Erziehungswissenschaft ist weder eine logische, noch eine in erster Linie politische Disziplin. Und so scheint denn dort mit dem Begriff der Homogenität höchst Verschiedenartiges auf: Während auf der einen Seite ein positiv besetzter Entwurf von Gleichheit als Ausgangspunkt aller Erziehungs- und Bildungsprozesse zugrunde gelegt wird, wird andererseits Gleichbehandlung als Problem angesehen. Besonders schulpädagogisch begegnet uns seit Langem eine kritische Kontextualisierung, in welcher die Vorstellung einer homogenen Lerngruppe nur mehr als überholt bezeichnet wird (vgl. Tillmann 2008; Trautmann und Wischer 2011), von der sich zu verabschieden dringend geboten scheint, gerade wegen der dadurch erzeugten Ungleichheiten. In anderen Zusammenhängen steht Gleichheit im Horizont von Normierung, oft untersucht und diskutiert im Zusammenhang mit Standardisierung der Lern- und Entwicklungserwartungen und der zunehmenden Institutionalisierung der Kindheit. Homogenität bzw. Homogenese wird also im Sinne von Gleichmachen kritisch diskutiert. Schließlich gehört zu den wichtigen Ideen der pädagogischen Moderne der Grundsatz, Chancen im Zugang zu Bildung und damit zu beruflichen Positionen seien nach Möglichkeit gleich zu verteilen bzw. zu gewähren. Diese Vielfalt der Bezugnahmen auf Homogenität und Gleichheit bilden die Hinterbühne der heutigen Debatten um Heterogenität und es soll diese Hinterbühne im Folgenden zur Vorderbühne werden. Ich unterscheide dabei drei Weisen der Thematisierung von Gleichheit und Homogenität, die in neueren erziehungswissenschaftlichen Debatten wirksam sind, jedoch meist nicht ausformuliert und transparent gehalten sind, und die zum Teil weit zurückreichende Traditionen haben (2). In einem nächsten Schritt werden dann die Inkommensurabilität der besprochenen Argumentationsmuster erläutert (3) und schließlich Desiderata für eine praxeologische kulturwissenschaftliche Forschung dargestellt (4). 


\section{Erziehungswissenschaftliche Thematisierung von Gleichheit und Homogenität}

\subsection{Die Bezugnahme auf Menschen- und bürgerliche Freiheitsrechte}

Die in der Erziehungswissenschaft zur Zeit vielleicht bekannteste Vertreterin dieses Ansatzes, Annedore Prengel, argumentiert auf dem Boden allgemeiner Menschenrechte, in denen alle, auch alle Kinder, als gleich angesehen werden hinsichtlich ihrer grundlegenden humanen Möglichkeiten, Bedürfnisse und Rechte. „So können“, schreibt Prengel, „mit guten Gründen alle Kinder als Gleiche hinsichtlich ihrer Kräfte im Sinne ästhetisch-humaner Möglichkeiten sowie hinsichtlich humaner Grundbedürfnisse nach Nahrung, Bindung an Bezugspersonen, nach Peer-Beziehungen, nach Bewegung und geistiger Anregung gelten“ (2014, 50).

Nur auf dem Boden dieser ursprünglichen Gleichheit als Ausgangspunkt aller Bildung und Erziehung könne dann nach Prengel eine pädagogische Konzeption von Heterogenität und Teilhabe entfaltet werden, die in Rücksichtnahme auf Prinzipien egalitärer Differenz einerseits auf Anerkennung der Vielfalt, andererseits aber auch auf Vermeidung von Kategorisierung zielt (vgl. ebd.). Damit ist zunächst die Anerkennung nicht-hierarchisierter Verschiedenheit im interpersonalen Verhältnis angesprochen. Im intrapersonalen Verhältnis geht es hingegen um die Berücksichtigung vielschichtiger Zugehörigkeiten im Sinne eines intersektionalen Ansatzes. Dadurch kann eine naive Identitätspolitik vermieden und die Gefahr der Positionierung der Einzelnen innerhalb bestimmter, determinierender Differenzkategorien verringert werden.

Geht man historisch einen Schritt zurück, so taucht ebenfalls mit Bezug auf die Menschen- und Bürgerrechte ab den 1960er und -70er Jahren die Normalisierungsdebatte auf, in der Anspruch und Hoffnung auf Angleichung der Lebensverhältnisse von Menschen mit Behinderungen im Sinne einer Normalisierung artikuliert wurden. Immer lauter und selbstverständlicher werden seitdem die Forderungen auf eine ebenbürtige Teilnahme an den alltäglichen Lebensverhältnissen von Menschen ohne Behinderung in allen Bereichen: Wohnen, Erziehung und Bildung, Arbeit, Politische Partizipation, Freizeit. In Deutschland formuliert Walter Thimm 1986 (zit. n. Mürner \& Sierck 2013, 70f. ): „Normalisierung stellt sich dar als ein Prozess, der schrittweise die Lebensbedingungen geistig behinderter Menschen [...] so gestaltet, dass ihr soziales Ansehen sowie ihre Fähigkeiten gefördert werden, sich möglichst selbständig mit den Lebensbedingungen auseinander zu setzen. Dieser Prozess zielt auf physische, soziale und personale Integration." Eine bestehende exkludierende Ungleichheit der Lebens- und Verwirklichungschancen soll so transformiert werden in eine inkludierende Normalität, die in Separierungspraktiken hervorgebrachte Differenzen durch Normalisierung minimiert. Von der Independent-Living-Bewegung bis zur heutigen Debatte um Inklusion finden diese Ansätze ihre Weiterentwicklung. 
Gleichheit steht also hier am Beginn und auf dem Boden aller Bildungsprozesse und fundiert zugleich das Ziel einer An-gleichung von Lebensverhältnissen und Verwirklichungschancen. Es wird somit als regulative Idee vorausgesetzt, was u.a. durch die angemessenen und ausreichenden Formen von Bildung und Erziehung als Realität erst hervorgebracht werden kann.

\subsection{Gleichheit der Chancen im Wettbewerb um gesellschaftliche Positionen}

In ganz anderer Weise wird der Gleichheitsbegriff verwendet in der Debatte um Chancengleichheit, die zu gewährleisten das Bildungswesen beansprucht sowie um die von erheblichen empirischen Forschungsbemühungen begleiteten Diskussionen um Gründe der Nicht-Realisierung dieses Anspruchs. Ausgangspunkt und Grundlage sind hier nicht die Menschen- und Bürgerrechte, sondern ist das moderne Prinzip der Leistung.

Dieses meritokratische Prinzip entwickelte sich seit dem ausgehenden 18. Jahrhundert als ein Instrument der Emanzipation der unteren Klassen (vgl. Reh \& Ricken 2018). Verteilung und Erreichbarkeit von sozialen und beruflichen Positionen sollte fortan von erbrachter Leistung des Individuums und nicht länger von Geburt in ein bestimmtes soziales Kollektiv geregelt werden. Nach und nach bedurfte es dazu auch der Installierung des auf diese beruflichen Positionen vorbereitenden Berechtigungswesens, einer Transformation des Schulwesens von einer Lehr- und Lerninstitution zu einer homogenisierte Qualifikationen vergebenden Institution. Dies brachte zunächst organisatorisch eine Reihe von Homogenisierungspraktiken mit sich - allerdings bei gleichzeitiger Beibehaltung bzw. Ausdifferenzierung des mehrgliedrigen Schulsystems in niedere und höhere Bildung sowie zunächst eindeutig geschlechtsspezifischen Zugangsregelungen. So wurde zum einen der Pflichtcharakter des Schulbesuchs verallgemeinert, es wurden nach und nach die Zugangs- und Abgangsregelungen vereinheitlicht und es wurden schließlich mit der allmählichen Durchsetzung der Jahrgangsklassen auch die Altersgruppen homogenisiert (vgl. Herrlitz, Hopf, Titze \& Cloer 2005; van Ackeren \& Klemm 2009).

Bis heute ist dieses Prinzip zum einen die Basisnorm und -legitimation für soziale Ungleichheit, zum anderen die Grundlage der Gleichbehandlung von Kindern und Jugendlichen in der Schule gemessen an gleichen Standarderwartungen der Lernresultate. Allerdings befindet sich die Tradition dieser auf Leistungsvergleichen basierenden Chancengleichheit heute in einem Widerstreit zwischen skeptischen Befragungen und ungebrochenem Optimismus ob seiner weiterhin geltenden Wirksamkeit und Tauglichkeit als Grundprinzip unseres Bildungs- und Berechtigungswesens.

Sensibilisiert durch die Diskussionen der 60er und 70er Jahre des 20. Jahrhunderts (vgl. Bourdieu und Passeron 1971; Dahrendorf 1965) sowie die Befunde der aktuellen internationalen Vergleichsstudien, richtet sich heute eine verstär- 
kte Aufmerksamkeit auf die Notwendigkeit, das Leistungsprinzip und die damit verbundene individualistische Ausrichtung der Leistungszuschreibung theoretisch und empirisch zu befragen. Wie eigentlich von wem festgelegt wird, was als eine gute oder weniger gute Leistung zu gelten hat, fragt z.B. Helmut Heid (2012); welche Implikationen in dem Konzept enthalten sind (z.B. Naturalisierung von Begabung) fragen Soziolog*innen wie Heike Solga (2005) und Andreas Hadjar (2008), und wie Schüler*innen dazu gelangen, sich selbst als leistungsstark, -willig, -motiviert oder auch leistungsschwach zu inszenieren, fragen - allerdings erst neuerdings - Schulethnograf*innen wie z.B. Georg Breidenstein (2012) oder Kerstin Rabenstein et al. (Rabenstein, Reh, Steinwand, Breuer 2014).

Optimistisch hingegen halten viele Vertreter der quantitativ verfahrenden Bildungsforschung beinahe ungebrochen am meritokratischen Prinzip fest und nähren die Überzeugung, man könne doch Bildungsgerechtigkeit (der Begriff scheint denjenigen der Chancengleichheit abzulösen) herstellen, wenn nur intensiv genug an den richtigen Stellen und auf die richtige Art und Weise die herkunftsbedingten Benachteiligungen im Sinne einer kompensatorischen Erziehung ausgeglichen würden. Allerdings ist damit auch die Pflicht des dazu unhinterfragt fähigen Individuums verbunden, diese Gaben der Gerechtigkeit in angemessener Weise zu nutzen und zu mehren.

Wie in bildungspolitisch einflussreichen Kontexten Bildungsgerechtigkeit gedacht wird, ist etwa den Veröffentlichungen des Aktionsrates Bildung zu entnehmen:

„Wenn [...] Bildungsangebote zur Herstellung von Bildungsgerechtigkeit von den Bedarfsträgern oder ihren Eltern nicht genutzt werden, wenn der Schulbesuch unregelmäßig ist, wenn die Lerner nicht alles dafür geben, die Lernangebote auch zu internalisieren, wenn sie den Lernerfolg anderer dadurch gefährden, dass sie sie am Lernen hindern, durch banale Unterrichtsstörung, durch die Diskriminierung von Leistung oder durch Leistungsbetrug, dann sind dieses Verhaltensweisen, die eine Gesellschaft nicht dulden kann, die erhebliche Mittel zur Herstellung von Bildungsgerechtigkeit investiert" (vbw 2007, 20f.).

Bildung wird hier in Hinsicht auf seine ökonomische Struktur, nämlich als Investition und damit Kostenverursacher für die im Besitz befindlichen, dimensioniert. Dieses Angebot der Besitzenden muss aber von den Individuen entsprechend genutzt werden; sie verhalten sich sonst gegenüber den Anbietern unsolidarisch, die auf die Freiheit verzichten, ihr Geld anders zu verwenden; ebenso verhielten sie sich auch gegenüber der Gesamtgesellschaft unsolidarisch, die erwarten kann, dass durch die Ermöglichung von Chancengleichheit auch das gemeinsame Ziel, nämlich einer Anhebung des Leistungsniveaus von allen ernsthaft verfolgt wird. Gegenüber dem zuvor Beschriebenen begegnen uns hier also andere Kontextualisierungen von Gleichheit: Chancengleichheit gilt als herzustellender Ausgangspunkt curricularen Lernens. Dieser ist notwendig, um dann 
eine Gleichbehandlung nach dem Leistungsprinzip legitimieren zu können. Nach dem Prinzip der Bedürfnisgerechtigkeit als einer besonderen Form der Distributivgerechtigkeit (vgl. Hübner 2014) müssen dafür die Ressourcen von Bildung, z.B. in Form von früher Sprachförderung, Bildungs- und Teilhabepaketen oder sonderpädagogischer Förderung verteilt werden. Es geht dabei aber nicht allein um die bedürfnisgerechte Verteilung von Bildungsangeboten, sondern zugleich auch um die Anerkennung des Leistungsprinzips durch die Empfänger, um eine kulturelle Homogenisierung mithin, deren ,Diskriminierung' nicht geduldet werden kann. Distributive Gerechtigkeit erfolgt hier nicht um der Erhöhung von Verwirklichungschancen der Bildungsbenachteiligten willen, denn dann müsste ja auch eine Entscheidung gegen das Leistungsprinzip denkbar sein, sondern folgt dem Ziel der Bildungsrenditensteigerung.

Das meritokratische Prinzip und die damit verbundene Etablierung allgemeiner Schulpflicht und weiterer schulorganisatorischer Homogenisierungsverfahren entstanden historisch in einer Doppelbewegung gemeinsam mit den europäischen Nationalstaaten. In der historischen Forschung gilt das allgemeine Schulwesen als diejenige Instanz, die im 19. Jahrhundert durch eine sprachliche, habituelle und kulturelle Homogenisierung der Schülerinnen und Schüler zu Nationalstaatlichkeit im Sinne einer ,citizenship education' wesentlich beigetragen hat (vgl. Coulmas 1985; Knabe 2000). Ebenso gilt in der Kindheits- und Jugendforschung die Scholarisierung als derjenige moderne Prozess, der durch Altershomogenisierung und eine flächendeckende Etablierung eines Bildungsmoratoriums die Phasen von Kindheit und Jugend in ihrer modernen Gestalt erst hervorgebracht hat.

\subsection{Kritik der Homogenese im kindheitssoziologischen Diskurs}

In kindheitswissenschaftlichen Zusammenhängen wird Homogenisierung mit den Begriffen der Normierung und Normalisierung untersucht (vgl. Kelle \& Tervooren 2008; Kelle \& Mierendorff 2013) und kritisch reflektiert. Diese Untersuchungen folgen auf eine Auseinandersetzung mit poststrukturalistischen Analysen einer zunehmend disziplinierten und institutionalisierten modernen Biografie sensu Foucault, sowie auch der neueren machtsensiblen Kindheitssoziologie (etwa Alanen und Mayall 2001; Sgritta 1987). Hier wird Homogenisierung daraufhin befragt, wie sie zu immer neuen Konstruktionen des Nicht-Normalen, der Norm-Abweichung führt, die durch die Zuschreibung von A-Normalität zu Erfahrungen der Exklusion und Repression führen können. Helga Kelle und ihre Arbeitsgruppe haben in diesem Zusammenhang außerschulische Orte der medizinischen Betreuung von Kindern untersucht und dabei die Ambivalenz von Standardisierung herausgearbeitet (vgl. Kelle 2010; auch Stechow 2004). Die Standarderwartungen an Körpermerkmale, Lernresultate und Verhaltensweisen ermöglichen, so die legitimierenden Hoffnungen, auf der einen Seite die rechtzeitige Intervention, erzeugen auf der anderen Seite aber immer auch Exklusionsme- 
chanismen. Kelle hat dabei ein erneutes Erstarken des Entwicklungsparadigmas mitsamt seiner normierenden und exkludierenden Mechanismen rekonstruiert. Schulpädagogisch rekonstruierte Wenning (2001) die Durchsetzung einer Normalität schulischer Leistungserwartungen in drei Schritten:

Zunächst erfolgt eine Homogenisierung der beteiligten Personen durch Gleichstellung, etwa durch Festsetzung einer Altersgrenze und Merkmalsbeschreibung von Eingangsvoraussetzungen eines Bildungsgangs. Anschließend wird der Maßstab zur Bewertung der individuellen Leistung (z.B. in einem Punktesystem) festgelegt und quantifiziert. Schließlich erfolgt eine Normalisierung mit der Bestimmung des Durchschnitts, d.h. einer Errichtung einer Minimalschwelle der Normalität, jenseits derer das Anormale beginnt.

In diesem Argumentationskontext wird Homogenese als Herstellung und Homogenität als Resultat des Erziehungs- und Sozialisationsprozesses untersucht, nun allerdings in der kritischen Perspektive poststrukturalistischen Denkens und in seiner zunehmenden Gefahr der Negierung von Pluralität. Diese von Foucault begonnene und in der Erziehungswissenschaft weiter entwickelte Kritik sensibilisiert für nicht intendierte Effekte der Homogenisierung und lässt gouvernementalitätstheoretisch die Frage nach einem Strukturwandel der Kindheit durch „neue Verfahren, Techniken und Methoden in Familien-, Kinder- und Jugendhilfe sowie Bildungs- und Betreuungseinrichtungen" entstehen, die in ihren enger werdenden Verknüpfungen „einen Gestaltwandel der Normalisierung von Kindheit“ (Kelle 2010, 31) hervorbringen.

\section{Die Inkommensurabilität der Argumentationsweisen}

Die drei eben skizzierten Argumentationskontexte stehen in einer unauflöslichen Spannung zueinander. Weder ließe sich eine von ihnen ignorieren, noch lassen sie sich ineinander auflösen oder hierarchisieren. Ebenso unscharf und vielgestaltig wie Begriff und Phänomen der Heterogenität (vgl. Budde 2013) wirkt sich derjenige der Homogenität auf aktuelle bildungswissenschaftliche Diskussionen aus.

Die drei vorgestellten Kontexte rekurrieren zunächst auf unterschiedliche Bildungsbegriffe. Wenn Bildung als die erworbene und lebenslang weiter zu entwickelnde Fähigkeit zu selbständiger und möglichst selbstbestimmter Auseinandersetzung mit dem eigenen Selbst, der Welt und den Anderen aufgefasst wird, fragt man unter Gleichheitsaspekten, wie eine solche Fähigkeit für Alle in ähnlicher Weise ermöglicht werden kann. Wenn andererseits Bildung im Sinne von Qualifikationen als (knappes) Gut, als kulturelles Kapital aufgefasst wird, das Auskunft zu geben verspricht über die gezeigten Leistungen seiner Besitzer, wird sie unter dem Gesichtspunkt der Distributivgerechtigkeit betrachtet und damit in einen gänzlich anderen Horizont gestellt. 
Die Argumentationskontexte thematisieren zweitens verschiedene Einsetzungspunkte von Gleichheit und Homogenität: Sie können sowohl als Ausgangspunkt im Sinne der Personenanerkennung, als auch als Resultat im Sinne der Wettbewerbsfitness nach kompensatorischer Erziehung, ebenso als Resultat im Sinne gleich befähigender Teilhabechancen oder als Prozess der Herstellung von normierender Standardisierung thematisiert werden. Jedes Mal gelangen dann andere Prozesse und Phänomene in den Blick, entstehen auch andere Zielvorstellungen für (künftige) pädagogische Handlungskonzepte.

Drittens ist für die weitere erziehungswissenschaftliche Forschung vielleicht am folgenreichsten, dass eine dauernde Verschränkung normativ ethischer Thematisierungen von Gleichheitsansprüchen mit deskriptiv kulturellen Analysen von Homogenisierung stattfindet. Beide Ebenen sind nur analytisch voneinander zu trennen, sowohl in praktischen als auch in erziehungswissenschaftlichen und bildungspolitischen Debatten ist meist beides miteinander verwoben. Besonders deutlich wird dies vielleicht in dem oben an zweiter Stelle vorgestellten historischen Kontext der Entstehung des meritokratischen Prinzips in Verschränkung mit derjenigen europäischer Nationalstaaten und der damit einhergehenden kulturellen Homogenisierung der Schüler*innen und der Institutionalisierung von Kindheit und Jugend in einem bis dahin nicht gekannten Maße. Man kann diese Verwobenheiten von Gleichheitsargumenten, die auf konkurrierende Gerechtigkeitsvorstellungen bezogen sind, mit Homogenitätsbeschreibungen, die auf kulturelle Entwicklungen bezogenen sind, systematisch für ein Ärgernis halten, man kann es aber auch als systematische Herausforderung annehmen.

Christoph Menke (2004, 22ff.) sieht die für die Moderne unhintergehbare Idee von Gleichheit relational zur Idee der Individualität und spricht hier von einer intern reflexiven Verfasstheit der Gleichheit. Die für die Moderne vorrangige Idee der Gleichheit enthält nach Menke in sich immer schon den doppelten Bezug auf das Allgemeine einerseits, das Individuelle andererseits.

Menke unterscheidet zunächst eine horizontale und eine vertikale Dimensioniertheit des modernen Gleichheitsdenkens. In der moralisch bestimmten Horizontale verhalten wir uns je einzeln so zueinander, dass wir den Anderen als im Prinzip gleich anerkennen. In der rechtlich bestimmten Vertikale beanspruchen wir eine Gesetzgebung, die für alle in gleicher Weise gilt, und die im Prinzip alle als gleichwertig anerkennt. Daneben bzw. darin gibt es jedoch ein weiteres Prinzip, das in der Moderne dem erstgenannten nachgeordnet ist, nämlich dasjenige der Ungleichbehandlung aufgrund von eigentümlichen Individualitäten. Menke nennt hier beispielhaft Bereiche der Fürsorge, der Treue oder Freundschaft. Sein Anliegen ist es nun, die Brechungen des Gleichheitsbegriffs, die sich aus dieser Antinomie von Gleichheit und Individualität ergeben, nicht gegeneinander auszuspielen, sondern im Sinne einer internen Reflexivität von Gleichheit zu fassen. „Denn die normative Orientierung an Individualität, von der aus die Idee der Gleichheit von 
außen befragt wird, tritt im inneren Vollzug der Gleichheit selbst schon auf; wir beziehen uns auf sie, genauer: wir übernehmen diese andere normative Orientierung gerade auch dann, wenn es uns um Gleichheit geht" (Menke 2004, 28). Egalitarismus und Non-Egalitarismus sind insofern schon immer aufeinander bezogen und können nicht als dichotome Optionen diskutiert werden, sofern man die Praktiken der Herstellung von Gleichheit und Ungleichheit in die theoretischen Überlegungen mit einbezieht. Wenn wir Menschen gleich behandeln oder zu Gleichen oder zu weniger Ungleichen zu machen suchen, treten wir im Vollzug dessen dem Einzelnen als Individuum gegenüber und anerkennen einen eventuell besonderen Bedarf der Behandlung etwa an Fürsorge, Zuwendung, Förderung oder Rücksichtnahme. Zugleich aber anerkennen wir das allgemeine Humanum und dessen Vermögen der Vernunft, der Einbildungskraft, der Bildsamkeit sowie der Leidensfähigkeit und Bedürfnisgebundenheit etc. Diese interne relationale und reflexive Verfasstheit führt pädagogisch zu all jenen Dilemmata, die aus der pädagogischen Praxis hinlänglich bekannt sind und die Frage enthalten, wann die Anwendung eines vertikal gegebenen Gesetzes (z.B. der Gleichbehandlung in der Leistungsbewertung) im Lichte der horizontal solidarisch verankerten Anerkennung von Unterschiedlichkeit angemessen oder aber unangemessen ist. Theoretisch kann Homogenität dann lediglich gefasst werden als eine Fiktion, deren unterschiedliche Dimensionen sich intern und dauerhaft befragen: Die vergleichbare Ähnlichkeit der Chancen, die vergeben werden, die vergleichbare Ähnlichkeit menschlichen Vermögens und ihrer Bedürfnisse, mit denen alle in das Erziehungs- und Bildungssystem eintreten, die vergleichbare Ähnlichkeit der kulturellen Orientierungen wie z.B. der Sprache oder dem schweigsamen Zustimmen zum Leistungsprinzip, die für ein gegenseitiges Verstehen notwendig sind, lassen sich nicht zu einem harmonischen Gesamtbild von Gleichheit zusammenfügen, sondern erfordern die Berücksichtigung der situativ und prozedural je veränderlichen Gleichzeitigkeit von Gleichheit und Ungleichheit, Homogenität und Heterogenität. So eröffnet die theoretische Reflexion Zugang zu einem notwendig in qualitativer Methodologie aufzusuchenden Forschungsfeld, in welchem danach gefragt wird, wie sich im Vollzug pädagogischer Praxis die interne Relativität von Gleichheit realisiert. Denn neben der grundlagentheoretischen Arbeit an der Verhältnisbestimmung von Heterogenität und Homogenität, von hierarchisierter und egalitärer Differenz existiert immer schon eine Praxis pädagogischer Felder, in welcher Gleichheit unter den Akteuren sich ereignet. Wie sie sich ereignet und wie sich diese Ereignisse empirisch erforschen lassen, das interessiert mich nun im letzten Schritt. 


\section{Herausforderungen an eine praxeologische empirische Erforschung der Gleichheit}

In einem kulturwissenschaftlichen Verständnis von Lehr- und Lernprozessen (vgl. z.B. Mollenhauer 2008; Helsper 2000; Thompson, Jergus \& Breidenstein 2014) werden pädagogische Felder als Orte kultureller Bedeutungsproduktionen und -reproduktionen aufgefasst und interpretiert, an denen materielle Artefakte und raumzeitliche Ordnungen ebenso mitwirken wie explizite und implizite Regelwerke, Organisationsformen und Muster legitimer Interaktionen. Einerseits geht es darin immer um die organisierte Gestaltung des Generationenwechsels in Form einer Sicherung des ,kulturellen Erbes', andererseits leisten die jeweiligen Akteure in der eigensinnigen Form der Aneignung dessen auch Beiträge zu Neuinterpretation und Resignifizierung. Einem praxeologischen Kulturbegriff zufolge (vgl. Reckwitz 2000) stellt sich Kultur so immer erst in verschiedenen Praktiken eines aufeinander abgestimmten Handelns her, welches auf für alle Teilnehmer gültige Sinnsysteme oder Sinnordnungen bezogen ist. Diese Ordnungen zeigen sich vor allem in wiederkehrenden und routinierten Handlungen, die als eine allen geläufige Praxis gefasst werden. Mit diesem Praxisbegriff findet auch die Bedeutung performativer, in körperlichen und materialisierten Interaktionsformen enthaltenen Handlungsformen Beachtung. Daher richtet sich das Forschungsinteresse der kulturwissenschaftlichen Bildungsforschung auf unspektakuläre Alltagspraktiken, die nur im ethnografischen Zugang sichtbar werden. Ausgangspunkt ist somit zunächst ein Primat der Praxen, aus denen die symbolischen Ordnungen hervorgehen bzw. immer wieder neu im alltäglichen Tun hervorgebracht, bestätigt oder auch modifiziert werden. Mit diesem Ansatz soll nun ein zukünftiges Forschungsfeld, das in Komplementarität zur Doing-difference-Forschung die Doing-equality-Prozesse in pädagogischen Feldern in den Blick nimmt, skizziert werden. Es wird hier zunächst für den Bereich der (Grund-)Schule konzipiert, eine Modifikation für institutionelle Felder der Pädagogik der Frühen Kindheit müsste sich daran anschließen.

Die Herstellung von Gleichheit und Orientierung an Gleichheitsvorstellungen wird dabei als eine kulturelle Praxis aufgefasst, die sich in der empirischen pädagogischen Wirklichkeit niemals für sich, sondern immer in Relation mindestens zu Unterscheidungspraktiken sowie zu Subjektpositionen ereignet. Wenn Kinder einer Grundschulklasse darüber klagen, dass der Lehrer die Mädchen immer bevorzugt, dann nehmen bestimmte zum Sprechen befugte einzelne Kinder eine Geschlechterdifferenz wahr und zugleich vor und bringen sie im performativen Akt der Klage als im Verhältnis zur Erwartung der Gleichbehandlung different stehend zum Ausdruck. Und sie unterstellen dem adressierten Erwachsenen, dass dieser der Erwartung im Prinzip zustimmt. Wenn ich nun im Folgenden, wie 
auch schon im Theoretischen auf Praktiken der Homogenisierung fokussiere, dann geschieht das immer im Kontext dieser dreifachen Relationalität.

Mit Bourdieu könnte man hier von einer ,Illusio`sprechen (vgl. Bourdieu 1998). Mit Illusio ist nicht ein reines Trugbild gemeint, das, wenn es erst als solches erkannt wird, ohne weiteres abgeschafft und durch ein passenderes ersetzt werden könnte; vielmehr beschreibt die Illusio eine notwendige stillschweigende Übereinkunft mit den je geltenden Spielregeln in einem sozialen Feld, welche Involvement und Engagement für das Spiel zuallererst ermöglichen. Nur wenn alle Akteure in ähnlicher Weise daran glauben, dass das Spiel den Einsatz lohnt und sich entsprechend mit vollem Ernst dem Spiel widmen, kommt es zur gleichmäßigen Teilnahme. Die Illusio ist weder von namhaften Spielleitern in rationalem Kalkül erfunden, noch steht es jeder Schulklasse oder KiTa-Gruppe frei, sich eine solche Illusio zu erschaffen. Vielmehr ist sie Ergebnis eines praktischen Sinns der Teilnehmer, der sich bewährt hat, aber nicht verfügbar ist und daher während des Spiels auch nicht zur Disposition steht. Aus der Performativitätsforschung ist bekannt, dass dieses Einverständnis zwar vielleicht heimlich im Sinne von nicht-explizit und auch nicht explizierbar, erfolgt, dass es aber keineswegs blind und ohne resignifizierende, widerständige Kommentare und Sinnverschiebungen der Akteure zur Aufführung kommt. Dies gilt für alle Akteure, Kinder wie Erwachsene, Schüler*innen wie Lehrer*innen in gleicher Weise.

Die Frage, ob und in welcher Weise eine Illusio von Gleichheit und Homogenität in der Praxis des pädagogischen Feldes wirksam wird, wird damit zu einer empirischen. Einerseits ist schulischer Unterricht wie jedes pädagogische Gruppengeschehen auf ein Mindestmaß an Verhaltens-Homogenität angewiesen, um als Geschehen einer Gruppe zu funktionieren, die auf einen gemeinsamen Gegenstand gerichtet ist und deren Lernresultate interindividuell aufeinander bezogen werden sollen; andererseits aber ist jedenfalls im Feld Schule diese notwendige Verhaltens-Ähnlichkeit ganz eng gebunden an die Herstellung einer Bühne zur Aufführung von Leistung, mit der eine andere Dimension von Homogenität ins Spiel kommt (vgl. Dietrich \& Wischmann 2014). Die kulturelle Notwendigkeit, sich an ähnlichen Situationsrahmungen zu orientieren - also etwa das Setting Morgenkreis oder mathematikbezogene Stillarbeit oder Sprachförderkurs - oder Differenzen zwischen solchen Rahmungen zu verhandeln, verschränkt sich mit der zu Zwecken der individuellen Bewertung vorgenommenen Vergleichbarkeit. In diesem allgemeinen Sinne sind kulturell angemessene und leistungsbezogen normativ geltende Prozesse der Homogenisierung daher zwar empirisch zu unterscheiden, jedoch im Folgeschritt auch wieder aufeinander zu beziehen. Es erläge sonst auch dieser Forschungsansatz der gleichen „struktur- und machttheoretischen Schwäche“, die Diehm et al. (2013, 35f.) angemahnt und in ihrem Ansatz ethnografischer Ungleichheitsforschung zu bearbeiten sich vorgenommen haben. Sie rekurrieren dabei auf den an Ethnografien gerichteten Vorwurf, durch 
die Mikroanalyse von Interaktionen würden diejenigen Strukturkategorien von Ungleichheit, die den Alltagsinteraktionen vorausliegen, verflüssigt oder unkenntlich, jedenfalls der kritischen Analyse nicht mehr zugänglich gemacht. Die gleiche Befürchtung kann man gegenüber einer komplementär verfahrenden Ethnografie der Gleichheit und Homogenität äußern, sofern diese sich unsensibel zeigt für die Differenz von alltagskulturell Sinn generierender und normativ Leistungen und Verhalten vergleichender und hierarchisierender Homogenität. Im praxeologischen Forschungsansatz geht man dennoch nicht davon aus, dass hier theoretische oder organisatorische Vorhaben bzw. gleichheitstheoretische Orientierungen in eine pädagogische Praxis umgesetzt werden, sondern nimmt vielmehr in Übereinstimmung mit Theorien zur Performativität sozialer Praxen an, dass sowohl kulturelle Traditionen der Homogenese reproduziert werden als auch in körperlichen, ludischen, interaktionistischen Dimensionen des Alltagshandelns entstehende Eigendynamiken des Feldes wirksam werden.

Gegenstand empirischer Untersuchung sind daher zunächst Praktiken, mit denen Erwachsene und Kinder im alltäglichen Geschehen eine Homogenität in der Gruppe herstellen. Damit wird auf Ebene des pädagogischen Alltags einem Phänomen nachgegangen, das bisher überwiegend theoretisch, historisch und schulorganisatorisch untersucht worden ist. Zwei Felder der Alltagspraktiken sind hier besonders in den Blick zu nehmen, damit die impliziten homogenisierenden Ordnungen und deren je situativ wirksamen Kommentierungen durch die Akteure, also Kinder wie Erwachsene, rekonstruierbar sind: Zum einen untersuchen wir körperlich vollzogene Praktiken raumzeitlicher Bewegungsroutinen als soziale Choreografien, die die Kindergruppe zu einer Gruppe von Gleichen machen (vgl. Hewitt 2005; Dietrich \& Riepe 2019). Zum anderen untersuchen wir sprachlich-symbolischen Praktiken. In beiden Feldern bilden Kinder Habitate aus, die eine erfolgreiche Teilnahme am schulischen Geschehen, die aber auch schulisches Geschehen überhaupt erst ermöglichen. Es interessieren also die praktischen Aufführungen alltäglicher Formate von Homogenität, wie sie entweder von den Professionellen oder von den Kindern untereinander, im häufigsten Fall aber von beiden gemeinsam aufgeführt, d.h. eingefordert, modifiziert, kommentiert oder auch verweigert werden.

In beiden Dimensionen, den körperlich-räumlichen wie den sprachlichen, sollten die Alltagspraktiken nicht nur in ihren sozialen und individualisierenden, sondern ebenso in ihren ästhetischen, oder eher noch: aisthetischen Sinnordnungen rekonstruiert werden. So begegnen uns z.B. in den alltäglichen sprachlichen Formaten nicht nur verschiedene Kompetenzen, die man mit den Kategorien von Wortschatz, Syntax und Semantik vermessen kann, sondern ebenso verschiedene schulkulturspezifische sowie auch fachspezifische Redegattungen wie das ,Narrativ' oder das ,Argumentativ'. Diese werden in Kindergarten und Grundschule intensiv eingeübt. Nur indem sie mitüben, werden Kinder zu Teilnehmer*innen 
des jeweiligen Feldes (vgl. Nelson 2007; Dietrich 2019), und nur indem die kulturellen Homogenisierungsübungen von allen in täglicher Iteration anerkannt werden, können Kindergarten und Schule als bedeutsame kulturelle Veranstaltungen fungieren.

Durch die Identifizierung und Analyse homogenisierender Alltagspraktiken in Unterrichts- und Erziehungssituationen können dann auch Prozesse der Differenzierung nach Leistung, Verhalten, Ethnizität, Sprache, Geschlecht etc. besser verstehbar gemacht werden, kann also die bis jetzt als Hinterbühne fungierende Diskursformation wieder zur Vorderbühne gemacht werden. Indem man rekonstruiert, wie die jeweilig beteiligten Akteure sich auf Vorstellungen von Gleichheit und Individualität im Sinne von gerechter Gleichbehandlung beziehen und welche Weisen der Homogenisierung zur Ermöglichung (schul-)kultureller Sinngenerierung sie dafür nutzen, können Aussagen zur Logik und Eigensinnigkeit des pädagogischen Feldes generiert werden. Schließlich steht zu erwarten, dass eine praxeologisch fundierte pädagogische Forschung auch die grundsatztheoretischen Diskussionen um eine gerechte Gleichheit und Ungleichheit befruchten kann.

\section{Literatur}

Ackeren, I. van \& Klemm, K. (2009): Entstehung, Struktur, Steuerung des deutschen Schulsystems: eine Einführung. Wiesbaden.

Alanen, L. \& Mayall, B. (Hrsg.) (2001): Conceptualizing Child-Adult Relations. London, New York.

Breidenstein, G. (2012): Zeugnisnotenbesprechung. Zur Analyse der Praxis schulischer Leistungsbewertung. Pädagogische Fallanthologie. Bd. 12. Opladen.

Bourdieu, P. \& Passeron, J. C.(1971): Die Illusion der Chancengleichheit. Untersuchungen zur Soziologie des Bildungswesens am Beispiel Frankreichs. Stuttgart.

Bourdieu, P. (1998): Praktische Vernunft. Zur Theorie des Handelns, deutsche Erstausgabe Auflage. Frankfurt a.M.

Budde, J. (Hrsg.)(2013): Unscharfe Einsätze: (Re-)Produktion von Heterogenität im schulischen Feld. Wiesbaden.

Coulmas, F. (1985): Sprache und Staat. Studien zur Sprachplanung und Sprachpolitik. Berlin und New York.

Dahrendorf, R. (1965): Bildung ist Bürgerrecht. Plädoyer für eine aktive Bildungspolitik. Hamburg.

Diehm I. (2008): Kindergarten und Grundschule - Zur Strukturdifferenz zweier Erziehungs- und Bildungsinstitutionen. In: Helsper, W., Böhme, J. (Hrsg.). Handbuch der Schulforschung. 2., durchgesehene und erweiterte Auflage. Wiesbaden. S. 557-575.

Diehm, I., Kuhn, M. \& Machold, C. (2013): Ethnomethodologie und Ungleichheit? Methodologische Herausforderungen einer ethnographischen Differenzforschung. In J. Budde (Hrsg.): Unscharfe Einsätze. (Re-)Produktion von Heterogenität im schulischen Feld. Wiesbaden. S. 29-51.

Dietrich, C. (2019): Ins Sprechen kommen. In: Theoretische Zugänge zur Pädagogik der frühen Kindheit. Eine kritische Vergewisserung. Hg. v. Cornelie Dietrich, Ursula Stenger und Claus Stieve. Weinheim und Basel. Im Erscheinen

Dietrich, C. \& Riepe, V. (2019): Praktiken der Homogenisierung: Soziale Choreographien im Schulalltag. Unveröffentlichtes Manuskript, eingereicht im Januar 2019 ZfPaed, in Begutachtung.

Dietrich, C. \& Wischmann, A. (2014): Genese von Heterogenität im Fachunterricht. Ein Beitrag zur Kontextualisierung von Differenzierungspraktiken. Online unter:http://bildungsforschung.org/index. php/bildungsforschung/article/view/166. (30.08.2017) 
Drerup, H. \& Fölling, W. (Hrsg.)(2006.): Gleichheit und Gerechtigkeit. Pädagogische Revisionen. Dresden.

Hadjar, A. (2008): Meritokratie als Legitimationsprinzip. Die Entwicklung der Akzeptanz sozialer Ungleichheit im Zuge der Bildungsexpansion. Wiesbaden.

Heid, H. (2012): Der Beitrag des Leistungsprinzips zur Rechtfertigung sozialer Ungerechtigkeit. Vierteljahresschrift für Heilpädagogik und ihre Nachbargebiete (VHN), 81. Jg., S. 22-34.

Helsper, W. (2000): Wandel der Schulkultur. In: Zeitschrift für Erziehungswissenschaft 3 (1), S. $35-$ 60.

Herrlitz, H. G., Hopf, W., Titze, H. \& Cloer, E. (2006): Deutsche Schulgeschichte von 1800 bis zur Gegenwart: Eine Einführung. Weinheim.

Hewitt, A. (2005): Social Choreography. Ideology as performance in dance and everyday movement. Durham and London.

Hübner, D. (2014): Bildung und Gerechtigkeit: Philosophische Zugänge. In: F. Dietrich, M. Heinrich $\&$ N. Thieme, N. (Hrsg.): Bildungsgerechtigkeit jenseits von Chancengleichheit. Theoretische und empirische Ergänzungen und Alternativen zu ,PISA'. Wiesbaden. S. 35-55.

Kelle, H. (Hrsg.) (2010): Kinder unter Beobachtung. Kulturanalytische Studien zur pädiatrischen Entwicklungsdiagnostik. Opladen.

Kelle, H. \& Mierendorff, J. (Hrsg.)(2013): Normierung und Normalisierung der Kindheit. Weinheim.

Kelle, H. \& Tervooren, A. (Hrsg.)(2008): Ganz normale Kinder. Heterogenität und Standardisierung kindlicher Entwicklung. Weinheim.

Knabe, F. (2000): Sprachliche Minderheiten und nationale Schule in Preußen zwischen 1871 und 1933. Münster.

Koller, H.-C., Casale, R. \& Ricken, N. (Hrsg.)(2014): Heterogenität. Zur Konjunktur eines pädagogischen Konzepts. Paderborn.

Liebau, E. \& Zirfas, J. (Hrsg.)(2008): Ungerechtigkeit der Bildung - Bildung der Ungerechtigkeit. Opladen \& Farmington Hills.

Menke, C. (2004): Spiegelungen der Gleichheit. Politische Philosophie nach Adorno und Derrida. Frankfurt a.M.

Mollenhauer, K. (2008): Vergessene Zusammenhänge: Über Kultur und Erziehung. 7. Auflage. Weinheim und Basel.

Mürner, C. \& Sierck, U. (2013): Behinderung. Chronik eines Jahrhunderts. Bonn: Bundeszentrale für politische Bildung.

Nelson, K. (2007): Young Minds in Social Worlds. Experience, Meaning and Memory. London.

Pauer-Studer, H. (2000): Autonom leben. Reflexionen über Freiheit und Gleichheit. Frankfurt a.M.

Prengel, A. (2014): Heterogenität oder Lesearten von Gleichheit und Freiheit in der Bildung. In H.C. Koller, R. Casale \& N. Ricken (Hrsg.),Heterogenität. Zur Konjunktur eines pädagogischen Konzepts. Paderborn. S. 45-67.

Rabenstein, K., Reh, S., Steinwand, J. \& Breuer, A. (2014): Jahrgang und Entwicklung. Zur Konstruktion von Leistung in jahrgangsgemischten Lerngruppen. In B. Kleiner, N. Rose (Hrsg.): (Re-) Produktion von Ungleichheiten im Schulalltag. Opladen/Berlin/Toronto. S. 135-154.

Reckwitz, A. (2000): Transformation der Kulturtheorien. Weilerswist-Metternich.

Reh, S. \& Ricken, N. (Hrsg.)(2018): Leistung als Paradigma. Zur Entstehung und Transformation eines pädagogischen Konzepts. Wiesbaden.

Sgritta, G.B. (1987): Childhood: Normalization and project. The Sociology of Childhood, edited by J. Qvortrup, International Journal of Sociology, 17(3), S. 38-57.

Solga, H. (2005): Meritokratie - die moderne Legitimation ungleicher Bildungschancen. In P.A. Berger \& H. Kahlert (Hrsg.): Institutionalisierte Ungleichheiten. Wie das Bildungswesen Chancen blockiert. München. S. 19-38. 
Stechow, E. von (2004): Erziehung zur Normalität. Eine Geschichte der Ordnung und Normalisierung der Kindheit. Wiesbaden.

Thompson, C., Jergus, K. \& Breidenstein, G. (Hrsg.)(2014): Interferenzen Perspektiven kulturwissenschaftlicher Bildungsforschung. Weilerswist-Metternich.

Tillmann, K.-J. (2008): Die homogene Lerngruppe - oder: System jagt Fiktion. In H.U. Otto \& T. Rauschenbach (Hrsg.). Die andere Seite der Bildung. Zum Verhältnis von formellen und informellen Bildungsprozessen. 2. Aufl.. Wiesbaden. S. 33-39.

Trautmann, M. \& Wischer, B. (2011): Heterogenität in der Schule. Eine kritische Einführung. Wiesbaden.

vbw - Vereinigung der Bayrischen Wirtschaft e. V. (Hrsg.) (2007): Bildungsgerechtigkeit. Jahresgutachten 2007. Wiesbaden.

Wenning, N. (2001): Differenz durch Normalisierung. In H. Lutz \& N. Wenning (Hrsg.). Unterschiedlich verschieden. Differenz in der Erziehungswissenschaft. Opladen. S. 275-295. 